\title{
Supersymmetric asymptotic safety is not guaranteed
}

\author{
Kenneth Intriligator ${ }^{a}$ and Francesco Sannino ${ }^{b}$ \\ ${ }^{a}$ Department of Physics, University of California, \\ San Diego, La Jolla, CA 92093 U.S.A. \\ ${ }^{b} C P^{3}$-Origins $\& 3$ the Danish Institute for Advanced Study, Danish IAS, \\ Univ. of Southern Denmark, Campusvej 55, DK-5230 Odense, Denmark \\ E-mail: keni@ucsd.edu, sannino@cp3-origins.net
}

ABSTRACT: It was recently shown that certain perturbatively accessible, nonsupersymmetric gauge-Yukawa theories have UV asymptotic safety, without asymptotic freedom: the UV theory is an interacting RG fixed point, and the IR theory is free. We here investigate the possibility of asymptotic safety in supersymmetric theories, and use unitarity bounds, and the a-theorem, to rule it out in broad classes of theories. The arguments apply without assuming perturbation theory. Therefore, the UV completion of a non-asymptotically free susy theory must have additional, non-obvious degrees of freedom, such as those of an asymptotically free (perhaps magnetic dual) extension.

KEYwords: Supersymmetry and Duality, Supersymmetric gauge theory

ArXiv EPrint: 1508.07411 


\section{Contents}

$\begin{array}{llr}1 & \text { Introduction } & 1\end{array}$

$2 \mathcal{N}=0$ asymptotic safety, brief review of $[1] \quad 2$

3 Supersymmetric RG fixed points and RG flows 4

4 Supersymmetric QCD with $N_{f}>3 N_{c}$ is unsafe $\quad 5$

5 Susy theories with general gauge group and matter $\quad 8$

6 Conclusions $\quad 9$

\section{Introduction}

The discovery of the Higgs crowns the Standard Model as one of the most successful theories of Nature. Researchers have been desperately seeking hints of possible BSM extensions of the Higgs sector. Gauge-Yukawa theories are backbone of the Higgs sector, and it is therefore crucial to thoroughly investigate all possibilities for their dynamics.

Recently, a surprise was found. One can consider a gauge theory with too many matter fields to be asymptotically free in the UV, so it is instead infrared free. Perturbation theory suggests that the theory is UV unsafe (the Landau pole), requiring a UV cutoff or completion. A Yukawa interaction for the matter of this theory, on it's own, would also be IR-free and UV-unsafe. Taken together, however, at least for a range of colors and flavors, the individually unsafe gauge and Yukawa interactions can cure each other and combine together to lead to a perturbatively accessible, fully interacting, non-supersymmetric, RG fixed point in the ultraviolet [1]. See [1,2] for the possibility that IR-free gauge and matter theory could have a UV fixed point even without the Yukawa interactions, albeit beyond perturbation theory.

The phenomenon of UV asymptotic safety opens the door to new model building possibilities [3, 4], and provide a novel playground to explore, e.g. the vacuum structure [5] and the thermodynamics of a new kind of matter [6]. The theories are different from the time-honoured case of complete asymptotic freedom [7-11], where the interactions instead shut off in the UV; see $[12,13]$ for recent studies.

Supersymmetry provides additional tools to explore the phases of gauge theories, beyond perturbation theory. For example, in $\mathcal{N}=1$ duals, two UV asymptotically free theories can RG flow to the same IR SCFT, or an IR-free theory can be UV completed to an asymptotically free UV dual, with different gauge group and matter content [14] (see e.g. [15] for a review). In this latter case, the IR free theory avoids the Landau pole 


\begin{tabular}{|c|c|ccc|}
\hline Fields & {$\left[\mathrm{SU}\left(N_{c}\right)\right]$} & $S U_{L}\left(N_{f}\right)$ & $S U_{R}\left(N_{f}\right)$ & $U_{V}(1)$ \\
\hline$A_{\mu}$ & $\operatorname{Adj}$ & 1 & 1 & 0 \\
$q$ & $\square$ & $\square$ & 1 & 1 \\
$\widetilde{q}$ & $\bar{\square}$ & 1 & $\square$ & -1 \\
$H$ & 1 & $\square$ & $\bar{\square}$ & 0 \\
\hline
\end{tabular}

Table 1. Field content of the $\mathcal{N}=0$ theory and field. The $A_{\mu}$ are the gauge fields, $q$ and $\tilde{q}$ are Weyl spinors in the $(1 / 2,0)$ Lorentz representation, and the $H$ are scalars.

by completing to a different free theory in the UV; this is not the same as interacting asymptotic safety.

It is interesting to investigate if asymptotic safety can also occur in supersymmetric theories - both for model building and for better understanding the phases of gauge theories. We will here rule out asymptotic safety for broad classes of supersymmetric theories, including $\mathcal{N}=1$ cousins of the nonsupersymmetric asymptotically safe theories [1]. Our methods and results do not rely on perturbation theory. In some cases, a hypothetical asymptotically safe UV fixed point would violate unitarity bounds [16], and in other cases it would violate the $4 \mathrm{~d}$ a-theorem [17-23], which can be explored in the susy context via the connection to 't Hooft anomalies for the superconformal R-symmetry [24-31].

The outline of this paper is as follows. In section 2, we summarize the perturbatively accessible, non-supersymmetric asymptotically safe case [1]. In section 3, we summarize the main susy-based methods that we will use in the following sections. In section 4 , we consider the direct $\mathcal{N}=1$ cousin of the theory considered in [1], based on $\mathcal{N}=1$ SQCD above the asymptotic freedom bound, $N_{f}>3 N_{c}$. We show that there cannot be a UV-interacting RG fixed point, neither with added gauge singlets Yukawa-coupled to the matter, nor for SQCD without the gauge singlets. In section 5 we apply susy-based methods to rule out asymptotically safe UV fixed points for theories with more general gauge group and matter content. We offer our conclusions in section 6 .

\section{$2 \mathcal{N}=0$ asymptotic safety, brief review of $[1]$}

Consider a massless theory with $\mathrm{SU}\left(N_{c}\right)$ gauge group and $N_{f}, \mathrm{SU}\left(N_{c}\right)$ fundamental, Dirac fermions $q_{D}$; we also write them as Weyl fermions $q$ and $\tilde{q}$. The theory has a global $\mathrm{SU}\left(N_{f}\right) \times \mathrm{SU}\left(N_{f}\right) \times \mathrm{U}(1)_{B}$ symmetry, and we include $N_{f} \times N_{f}$ complex scalar fields, which are gauge singlets. The matter content is summarized in table I. The Lagrangian is

$$
\begin{aligned}
L=-\frac{1}{4 g^{2}} \operatorname{Tr} F^{\mu \nu} F_{\mu \nu}+\operatorname{Tr}\left(\bar{q}_{D} i \not D q_{D}\right)+\operatorname{Tr}\left(\partial_{\mu} H^{\dagger} \partial^{\mu} H\right) \\
+y \operatorname{Tr}(\widetilde{q} H q+\text { h.c. })-h \operatorname{Tr}\left(H^{\dagger} H\right)^{2}-v\left(\operatorname{Tr} H^{\dagger} H\right)^{2},
\end{aligned}
$$

and $\mathrm{Tr}$ indicates the appropriate trace over the suppressed color and flavor indices. The classical theory is scale invariant with four marginal couplings: the gauge coupling $g$, the Yukawa coupling $y$, the quartic scalar couplings $h$ and the double-trace scalar coupling $v$. 
In the quantum theory, it is convenient to introduce

$$
\alpha_{g}=\frac{g^{2} N_{c}}{(4 \pi)^{2}}, \quad \alpha_{y}=\frac{y^{2} N_{c}}{(4 \pi)^{2}}, \quad \alpha_{h}=\frac{h N_{f}}{(4 \pi)^{2}}, \quad \alpha_{v}=\frac{v N_{f}^{2}}{(4 \pi)^{2}},
$$

with appropriate powers of $N_{c}$ and $N_{f}$ in the normalization to allow for the Veneziano limit of large $N_{c}$ and $N_{f}$, holding fixed

$$
x \equiv \frac{N_{f}}{N_{c}} \equiv \frac{11}{2}+\epsilon .
$$

The one-loop beta function is asymptotically free for $\epsilon<0$, and infrared free for $\epsilon>0$. In this notation, the usual Banks-Zaks [32] limit is $\epsilon$ infinitesimally negative, whereas [1] instead considers $\epsilon$ infinitesimally positive.

The relevant beta functions $\beta_{i}\left(\alpha_{g}, \alpha_{y}, \alpha_{h}, \alpha_{v}\right) \equiv \partial_{t} \alpha_{i}$ for each coupling $i=(g, y, h, v)$ of the theory (2.1) have been obtained in [33] in dimensional regularization using [34-37]. The point $\alpha_{i}=0$ is IR attractive, since none of the couplings are asymptotically free. As shown in [1], the beta functions vanish at non-zero couplings, compatible with classical and quantum scalar potential stability [5], given by

$$
\begin{aligned}
& \alpha_{g}^{*}=\frac{26}{57} \epsilon+\frac{23(75245-13068 \sqrt{23})}{370386} \epsilon^{2}+\mathcal{O}\left(\epsilon^{3}\right) \\
& \alpha_{y}^{*}=\frac{4}{19} \epsilon+\left(\frac{43549}{20577}-\frac{2300 \sqrt{23}}{6859}\right) \epsilon^{2}+\mathcal{O}\left(\epsilon^{3}\right) \\
& \alpha_{h}^{*}=\frac{\sqrt{23}-1}{19} \epsilon+\mathcal{O}\left(\epsilon^{2}\right), \\
& \alpha_{v}^{*}=\frac{1}{19}(-2 \sqrt{23}+\sqrt{20+6 \sqrt{23}}) \epsilon+\mathcal{O}\left(\epsilon^{2}\right) .
\end{aligned}
$$

The phase diagram of the theory was established in [1] at the next-to-leading order accuracy and extended to the next-to-next leading order in [5].

See figure 1 of [5] for the RG trajectories of the couplings $\left(\alpha_{g}, \alpha_{y}, \alpha_{h}, \alpha_{v}\right)$; the beta functions are all positive for $\alpha_{i=g, y, h, v}<\alpha_{i}^{*}$. The interesting RG trajectory goes from the interacting fixed point at $\alpha_{i}=\alpha_{i}^{*}$ in the UV, and ends at the free theory, $\alpha_{i}=0$ in the IR. Along this one-dimensional line of physics, in the $4 \mathrm{~d} \alpha_{i}$ space, the Yukawa and scalar quartic couplings are all determined in terms of the running gauge coupling. This dynamical relation among the couplings is dictated by the dimension of the critical surface. In the UV, the gauge coupling approaches the interacting, asymptotically safe, UV fixed point by a power-law in the RG scale

$$
\lim _{\mu / \mu_{0} \rightarrow \infty} \alpha_{g}(\mu) \rightarrow \alpha_{g}^{*}+\left(\alpha_{g}\left(\mu_{0}\right)-\alpha_{g}^{*}\right)\left(\frac{\mu}{\mu_{0}}\right)^{-\frac{104}{171} \epsilon^{2}+\mathcal{O}\left(\epsilon^{3}\right)},
$$

(see [5] for the all- $\mu$ running, in terms of the Lambert function $W(\mu)$ ).

It would interesting to extend the results beyond the perturbative regime via, for example, first principle lattice simulations [38-42]. An alternative limit is QCD for fixed $N_{c}$ and large $N_{f}$ where, for the theory without scalars, at leading order $1 / N_{f}$, an UV asymptotically safe fixed point seems also to appear $[1,2]$. Supersymmetry provides tools to explore beyond perturbation theory, as we will discuss in the following sections. 


\section{Supersymmetric RG fixed points and RG flows}

We focus on theories in $d=4$ spacetime dimensions, with $\mathcal{N}=1$ supersymmetry. A RG fixed point is an $\mathcal{N}=1$ superconformal field theory (SCFT), which necessarily has a conserved $\mathrm{U}(1)_{R}$ global symmetry. The $\mathrm{U}(1)_{R}$ current is in the same supermultiplet [43] as the energy-momentum tensor and the supercharge currents; this leads to many useful exact relations. For a unitary theory, the operators form unitary representations of the superconformal group, which implies that operator dimensions have various lower bounds. For example, regardless of supersymmetry, all gauge invariant spin $j=\bar{j}=0$ operators have the lower bound (generators act with implicit commutators) [16] (see also e.g. [44])

$$
D(\mathcal{O}) \geq 1, \quad D(\mathcal{O})=1 \leftrightarrow P_{\mu} P^{\mu}(\mathcal{O})=0,
$$

so the bound is saturated if and only if the operator $\mathcal{O}$ is a decoupled, free field. Chiral primary operators have dimension, $D$, and superconformal $\mathrm{U}(1)_{R}$ charge, $R$, related by

$$
D(\mathcal{O})=\frac{3}{2} R(\mathcal{O})
$$

In particular, using (3.2) for the matter chiral superfields $Q_{i}$ of a supersymmetric gauge theory relates the matter anomalous dimensions $\gamma_{i}$ to their superconformal $\mathrm{U}(1)_{R}$ charge.

$$
D\left(Q_{i}\right) \equiv 1+\frac{1}{2} \gamma_{i}(g)=\frac{3}{2} R\left(Q_{i}\right) \equiv \frac{3}{2} R_{i} .
$$

The conformal anomaly $a$ of the SCFT is exactly given by the superconformal $\mathrm{U}(1)_{R}$ 't Hooft anomalies $[24,25]$ (we rescale the overall normalization factor for convenience)

$$
a(R)=3 \operatorname{Tr} \mathrm{U}(1)_{R}^{3}-\operatorname{Tr} \mathrm{U}(1)_{R} .
$$

For a gauge theory with gauge group, $G$, and matter fields $Q_{i}$, in representations $r_{i}$ of $G$, the 't Hooft anomalies evaluate to

$$
a\left(R_{i}\right)=2|G|+\sum_{i}\left|r_{i}\right| a_{1}\left(R_{i}\right)
$$

where $|G|=\left|r_{\text {Adjoint }}\right|$ is the number of generators of the gauge group, $\left|r_{i}\right|$ is the dimension of the $r_{i}$ representation, $R_{i} \equiv R\left(Q_{i}\right)$ is the $\mathrm{U}(1)_{R}$ charge of $Q_{i}$, and we define the function

$$
a_{1}(R) \equiv 3(R-1)^{3}-(R-1) .
$$

Among all possible, conserved $\mathrm{U}(1)_{R}$ symmetries, the superconformal $\mathrm{U}(1)_{R}$ is that which maximizes $a(R)$ [26]. For example, for a chiral superfield $X$ of charge $R(X)=R$ (so $R\left(\psi_{X}\right)=R-1$ ), the function is $a(R)=a_{1}(R)$ in (3.6). The function $a_{1}(R)$ has a local maximum at the free-field value, $R=\frac{2}{3}$, and a local minimum at $R=\frac{4}{3}$. Indeed $a_{1}(R)=-a_{1}(2-R)$, so $a_{1}(R=1)=0$, fitting with massive operators contributing $a=0$. Note also that $a_{1}(R)$ is below the local maximum, $a_{1}(R)<a_{1}(R=2 / 3)$, for all $R$ in the range $R<5 / 3$ (see [48] for a related conjectured phase diagnostic). For unconstrained, i.e. free chiral superfields, we maximize the function (3.6) to get $R_{*}=2 / 3$, the free-field 


\begin{tabular}{|c|c|cccc|}
\hline Fields & {$\left[\mathrm{SU}\left(N_{c}\right)\right]$} & $S U_{L}\left(N_{f}\right)$ & $S U_{R}\left(N_{f}\right)$ & $U_{V}(1)$ & $\mathrm{U}(1)_{R}$ \\
\hline$W_{\alpha}$ & $\operatorname{Adj}$ & 1 & 1 & 0 & 1 \\
$Q$ & $\square$ & $\overline{1}$ & 1 & 1 & $1-\frac{N_{c}}{N_{f}}$ \\
$\widetilde{Q}$ & $\overline{1}$ & 1 & $\square$ & -1 & $1-\frac{N_{c}}{N_{f}}$ \\
$H$ & 1 & $\square$ & $\bar{\square}$ & 0 & $2 \frac{N_{c}}{N_{f}}$ \\
\hline
\end{tabular}

Table 2. The $\mathcal{N}=1$ superfield content, cousins of the theory (1). $W_{\alpha}$ is the gauge vector superfield, $Q$ and $\widetilde{Q}$ are the matter chiral superfields, and $H$ are gauge singlet chiral superfields.

value of the R-charge, corresponding to $D(X)=1$. With interactions, we maximize $a(R)$ subject to the constraint that the interactions do not violate the R-symmetry. Accidental symmetries affect a-maximization [28](see also e.g. [29]), which if present leads to a larger value of $a$.

Away from a RG fixed point, the beta functions are proportional to how the couplings break the superconformal $\mathrm{U}(1)_{R}$. The gauge coupling beta function is proportional to the ABJ triangle anomaly of the $\mathrm{U}(1)_{R}$ current with two $G$ gauge fields, i.e. $\operatorname{Tr} G^{2} \mathrm{U}(1)_{R}$ :

$$
\beta(g)=-\frac{3 g^{3}}{16 \pi^{2}} f\left(g^{2}\right) \operatorname{Tr} G^{2} \mathrm{U}(1)_{R}, \quad \operatorname{Tr} G^{2} \mathrm{U}(1)_{R}=T(G)+\sum_{i} T\left(r_{i}\right)\left(R_{i}-1\right) .
$$

Our normalization for the quadratic Casimir of the adjoint $T(G)$ is $T\left(\mathrm{SU}\left(N_{c}\right)\right)=N_{c}$, so the fundamental representation of $\mathrm{SU}(N)$ has $T\left(r_{\text {fund }}\right)=\frac{1}{2}$. The function $f\left(g^{2}\right)=1+\mathcal{O}\left(g^{2}\right)$ is scheme dependent (and presumed positive). Using (3.3) gives the statement of the NSVZ exact beta function (NSVZ also have a favored, specific scheme choice for $f\left(g^{2}\right)$ ) [45]:

$$
\beta\left(8 \pi^{2} g^{-2}\right)=f\left(g^{2}\right)\left(3 T(G)-\sum_{i} T\left(r_{i}\right)\left(1-\gamma_{i}(g)\right)\right)
$$

For superpotential terms $W_{y}$, the holomorphic coupling $y$ prefactor has beta function

$$
\beta(y)=\frac{3}{2} y\left(R\left(W_{y}\right)-2\right) .
$$

\section{Supersymmetric QCD with $N_{f}>3 N_{c}$ is unsafe}

As a first class of examples, we consider the $\mathcal{N}=1$ cousin of the theory (2.1), with superfield content and quantum symmetries summarized in table 2. The superpotential is:

$$
W=y \operatorname{Tr} Q H \widetilde{Q},
$$

with $y$ the Yukawa coupling and Tr contracts the implicit gauge and flavor indices. The quartic in $H$ interactions in (2.1) are incompatible with holomorphy of the superpotential (and the $\mathrm{SU}\left(N_{f}\right) \times \mathrm{SU}\left(N_{f}\right)$ global symmetry does not allow for a holomorphic variant). 
Taking $N_{c}$ and $N_{f}$ large, and properly rescaling the couplings as

$$
\alpha_{g} \equiv \frac{g^{2} N_{c}}{(4 \pi)^{2}}, \quad \alpha_{y} \equiv \frac{y^{2} N_{c}}{(4 \pi)^{2}},
$$

the two loops beta functions are, dropping terms subleading in $1 / N_{c}$,

$$
\begin{aligned}
& \beta\left(\alpha_{g}\right) \approx-2 \alpha_{g}^{2}\left[3-\frac{N_{f}}{N_{c}}+\left(6-4 \frac{N_{f}}{N_{c}}\right) \alpha_{g}+2 \frac{N_{f}^{2}}{N_{c}^{2}} \alpha_{y}+\mathcal{O}\left(\alpha^{2}\right)\right], \\
& \beta\left(\alpha_{y}\right) \approx 2 \alpha_{y}\left[\left(2 \frac{N_{f}}{N_{c}}+1\right) \alpha_{y}-2 \alpha_{g}+\mathcal{O}\left(\alpha^{2}\right)\right] .
\end{aligned}
$$

To connect with (3.8) and (3.9) note that they give (using (3.3), and $R(W)=R(H)+$ $2 R(Q))$

$$
\beta\left(\alpha_{g}\right)=-2 \alpha_{g}^{2} f\left(\alpha_{g}\right)\left(3-\frac{N_{f}}{N_{c}}\left(1-\gamma_{Q}\right)\right), \quad \beta\left(\alpha_{y}\right)=\alpha_{y}\left(\gamma_{H}+2 \gamma_{Q}\right),
$$

and to the relevant order (dropping $\mathcal{O}\left(\alpha_{g, y}^{2}\right)$ and $\mathcal{O}\left(1 / N_{c}\right)$ ) we have

$$
f\left(\alpha_{g}\right) \approx 1+2 \alpha_{g}, \quad \gamma_{Q} \approx-2 \alpha_{g}+2 \frac{N_{f}}{N_{c}} \alpha_{y}, \quad \gamma_{H} \approx 2 \alpha_{y}
$$

For $N_{f}>3 N_{c}$, the theory is not asymptotically free, and $\alpha_{g}=\alpha_{y}=0$ is IR-attractive. We first note that there cannot be a perturbative, interacting, UV-safe fixed point. Define $\epsilon \equiv N_{f} / N_{c}-3$, with $N_{f}$ and $N_{c}$ such that $0<\epsilon \ll 1$. The condition $\beta\left(\alpha_{y}\right)=0$ then gives $\alpha_{y} \approx \frac{2}{7} \alpha_{g}$, and then $\beta\left(\alpha_{g}\right)$, to leading order in small $\epsilon$, is

$$
\beta\left(\alpha_{g}\right) \approx 2 \alpha_{g}^{2}\left[\epsilon+\frac{6}{7} \alpha_{g}\right]
$$

so the relative sign is such that, for positive $\epsilon, \beta\left(\alpha_{g}\right) \neq 0$ unless $\alpha_{g}=0$.

We now argue, including at the non-perturbative level, that there cannot be a UVsafe interacting SCFT. We assume that the superconformal $\mathrm{U}(1)_{R}$ is not emergent or accidental, in which case it must be the anomaly free R-symmetry that is preserved by the superpotential, i.e. the $\mathrm{U}(1)_{R}$ given in table II; this ensures that the beta functions (4.4) vanish. The dimension of the operators $H$ are then determined to be

$$
D(H)=\frac{3}{2} R(H)=3 \frac{N_{c}}{N_{f}} .
$$

These statements are all correct for the IR SCFT fixed point when $N_{f}<3 N_{c}$. But, for $N_{f}>3 N_{c}$, (4.7) would violate the unitarity bound (3.1). This is impossible, since the original theory is unitary. There thus cannot be an interacting UV SCFT for $N_{f}>3 N_{c}$.

It follows from Seiberg duality [14] that the $N_{f}>3 N_{c}$ theory can actually be UVcompleted to an asymptotically free dual, rather than an interacting, UV-safe, SCFT.

A potential loophole in the above argument is that apparent unitarity bound violations mean that the corresponding field - in this case $H$ - is instead a free, decoupled field. 
So we set $\alpha_{y}=0$, which is equivalent to considering SQCD without the $H$ singlets. We now argue that SQCD without the $H$ fields also cannot have a UV safe RG fixed point for $N_{f}>3 N_{c}$. Consider first the perturbative regime, $\epsilon \equiv N_{f} / N_{c}-3$, with $0<\epsilon \ll 1$. Taking $\alpha_{y}=0$ in (4.3) gives

$$
\beta\left(\alpha_{g}\right) \approx 2 \alpha_{g}^{2}\left[\epsilon+6 \alpha_{g}\right]
$$

so $\beta\left(\alpha_{g}\right) \neq 0$ for $\epsilon>0$ and $\alpha_{g} \neq 0$, i.e. there is no perturbative UV-safe fixed point.

We now rule out asymptotic safety beyond perturbation theory. First note that, for $\alpha_{g} \neq 0$, having $\beta\left(\alpha_{g}\right)=0$ requires that the superconformal $\mathrm{U}(1)_{R}$ be that in table II, so

$$
D_{\mathrm{SCFT}}(Q)=D_{\mathrm{SCFT}}(\widetilde{Q}) \equiv 1+\frac{1}{2} \gamma_{Q}=\frac{3}{2} R_{\mathrm{SCFT}}(Q)=\frac{3}{2}\left(1-\frac{N_{c}}{N_{f}}\right) .
$$

The gauge invariant chiral operators, the mesons $\mathcal{M}=Q \tilde{Q}$ and baryons $\mathcal{B}=Q^{N_{c}}$ have

$$
\begin{aligned}
D_{\mathrm{SCFT}}(\mathcal{M}) & =\frac{3}{2} R_{\mathrm{SCFT}}(\mathcal{M})=3 \frac{N_{f}-N_{c}}{N_{f}}, \\
D_{\mathrm{SCFT}}(\mathcal{B}) & =D_{\mathrm{SCFT}}(\widetilde{\mathcal{B}})=\frac{3}{2} R_{\mathrm{SCFT}}(\mathcal{B})=\frac{3}{2} N_{c} \frac{N_{f}-N_{c}}{N_{f}} .
\end{aligned}
$$

These expressions are indeed correct for the IR fixed point of SQCD in the conformal window [14] $\frac{3}{2} N_{c}<N_{f}<3 N_{c}$. Extrapolating to the hypothetical, UV fixed point for $N_{f}>3 N_{c}$, these expressions would apply, and the operators would satisfy the unitarity bound (3.1).

We therefore rule out a hypothetical, interacting UV fixed point for $N_{f}>3 N_{c}$ SQCD on different grounds, by noting that it would violate the $4 \mathrm{~d} a$-theorem $[17,22,23]$,

$$
\Delta a \equiv a_{\mathrm{UV}}-a_{\mathrm{IR}}>0 .
$$

If the hypothetical UV-safe fixed point exists, its $a$ would be given by (3.5) with the interacting, anomaly free $\mathrm{U}(1)_{R}$, i.e. $R_{\mathrm{SCFT}}(Q)=\left(N_{f}-N_{c}\right) / N_{f}$,

$$
a_{\text {hypothetical UV }}=a_{\mathrm{SCFT}}=2\left(N_{c}^{2}-1\right)+2 N_{f} N_{c} a_{1}\left(R=1-\frac{N_{c}}{N_{f}}\right),
$$

with $a_{1}(R)$ the function (3.6). There would be a RG flow from the hypothetical UV SCFT to the IR-free theory with $\alpha_{g}=0$ and thus $R(Q)=2 / 3$ :

$$
a_{\mathrm{IR}}=a_{\text {free }}=2\left(N_{c}^{2}-1\right)+2 N_{f} N_{c} a_{1}(R=2 / 3)=2\left(N_{c}^{2}-1\right)+\frac{4}{9} N_{f} N_{c} .
$$

So this RG flow would violate the a-theorem (4.11):

$$
a_{\mathrm{UV}-\mathrm{safe}}-a_{\mathrm{IR}-\text { free }}=2 N_{c} N_{f}\left(a_{1}\left(R=1-\frac{N_{c}}{N_{f}}\right)-a_{1}(R=2 / 3)\right)<0
$$

where the inequality is evident from graphing $a_{1}(R)$ in (3.6) since, for $3 N_{c}<N_{f}<\infty$, the R-charge $R(Q)=1-\frac{N_{c}}{N_{f}}$ is in the range $\frac{2}{3}<R(Q)<1$, and $a_{1}$ in that range is below its local maximum $a_{1}(R=2 / 3)$. Given the wrong sign (4.14), we conclude that there cannot be an interacting, UV safe fixed point. Instead, IR-free electric SQCD theory can be UV-completed to an asymptotically free magnetic dual. For SQCD in the conformal window, the identification of the endpoints is opposite from that in (4.14) (free in the UV and interacting in the IR). Both those RG flows of course do satisfy the a-theorem [24, 25]. 


\section{Susy theories with general gauge group and matter}

We first consider susy gauge theories without superpotential terms, $W_{\text {tree }}=0$. The exact beta function for the gauge coupling is as in (3.7), and the condition $\beta(g)=0$ is equivalent to the condition that the superconformal $R_{\mathrm{SCFT}}\left(Q_{i}\right) \equiv R_{\mathrm{SCFT}, \mathrm{i}}$ is anomaly free:

$$
\begin{gathered}
\beta\left(\alpha_{g}\right)=0 \leftrightarrow T(G)+\sum_{i} T\left(r_{i}\right)\left(R_{i}-1\right)=0 \quad \leftrightarrow \quad 3 T(G)-\sum_{i} T\left(r_{i}\right)\left(1-\gamma_{Q_{i}}\right)=0 . \\
\text { So not asymptotically free has: } \quad 3 T(G)-\sum_{i} T\left(r_{i}\right)<0 .
\end{gathered}
$$

It is then impossible to satisfy (5.1) perturbatively, since all perturbative $\gamma_{Q_{i}}$ are negative.

Generalizing the argument of the previous section, we can moreover rule out UV-safe SCFTs in the range (5.2), without relying on perturbation theory. The RG flow from the hypothetical, asymptotically safe SCFT in the UV, to the free $\alpha_{g}=0$ theory in the IR, would violate the a-theorem. Using (3.5), the hypothetical flow has

$$
\Delta a=a_{\mathrm{UV}-\mathrm{safe}}-a_{\mathrm{IR}-\text { free }}=\sum_{\text {all matter } Q_{i}}\left|r_{i}\right|\left(a_{1}\left(R_{\mathrm{SCFT}, i}\right)-a_{1}\left(R_{i}=2 / 3\right)\right) .
$$

The general expression for $R_{\mathrm{SCFT}, i}$ follows from a-maximization [26], i.e. maximizing $a_{1}\left(R_{\mathrm{SCFT}, i}\right)$ over the $R_{i}$, subject to the constraint in (5.1). This gives [27],

$$
R_{\mathrm{SCFT}, i}=1-\frac{1}{3}\left(1+\frac{\lambda T\left(r_{i}\right)}{\left|r_{i}\right|}\right)^{1 / 2}
$$

where the Lagrange multiplier is determined via (5.1). In the asymptotically free case, this yields $\lambda=\left(g_{*}^{2} / 2 \pi^{2}\right)+\mathcal{O}\left(g_{*}^{4}\right)$ (the higher order terms are scheme dependent) [27, 30,31]. In the non-asymptotically free case (5.2), on the other hand, the constraint leads to $\lambda<0$, and all $R_{\mathrm{SCFT}, \mathrm{i}}$ would be in the range $2 / 3<R_{i}<1$. It is then clear from the graph of $a_{1}(R)$ that, since $R_{i}<5 / 3$, every term in $(5.3)$ is negative, $a_{1}\left(R_{\mathrm{SCFT}, i}\right)-a_{1}\left(R_{i}=2 / 3\right)<0$, the flow from the hypothetical, UV-safe fixed point would violate the a-theorem. So susy gauge theories with $W=0$ cannot be interacting UV-safe, and IR free, without some new element (for example, some accidental symmetry in the interacting UV theory).

We now consider adding superpotential terms. The upshot of a-maximization is that the microscopic fields have superconformal $R$-charges given by [30]

$$
R\left(Q_{i}\right)=1-\frac{\epsilon_{i}}{3} \sqrt{1-2 \gamma_{i}^{(1)}(\lambda)},
$$

where $\epsilon_{i}= \pm 1$ (see [30, 49] for curiosities related to $\epsilon_{i}$ sign changes in RG flows) and $\gamma_{i}^{(1)}$ are linear in the $\lambda$ and related to the one-loop anomalous dimensions. The values of $R_{\mathrm{SCFT}}\left(Q_{i}\right)$ are obtained from (5.5) by solving for the $\lambda_{\mathrm{SCFT}}$ such that all $R$-charge conservation constraints are satisfied, i.e. all gauge groups $G$ have $\operatorname{Tr} G^{2} \mathrm{U}(1)_{R}=0$ and all $W_{y}$ have $R\left(W_{y}\right)=2$, i.e. all beta functions (3.7) and (3.9) vanish. Note that all (5.5) yield $R_{\mathrm{SCFT}, \mathrm{i}}<4 / 3$, and thus all $a_{1}\left(R=R_{\mathrm{SCFT}, \mathrm{i}}\right)<a_{1}(R=2 / 3)$. The RG flow from a hypothetical UV-safe SCFT, to the IR-free theory, would have $\Delta a$ given by (5.3), which again would violate the a-theorem because every term in the sum has the wrong sign. 
Also, as in the examples (4.1), gauge singlets $H$ coupled to composite operators $\mathcal{O}$, of classical dimension $D_{c l}(\mathcal{O})=2$, leads to a unitarity bound problem for hypothetical UV-safe SCFTs. Because the theories are not asymptotically free, the condition (5.1) leads to $R_{\mathrm{SCFT}}(\mathcal{O})>R_{\text {free }}(\mathcal{O})$, and then $R(W)=2$ requires $R(H)<2 / 3$; then $D_{\mathrm{UV}-\text { safe }}=$ $\frac{3}{2} R(H)<1$, violating the unitarity bound. The $H$ fields must instead remain free; this fits with the fact that there is no interacting, UV-safe SCFT after all.

Note that $\mathcal{N}=4$ supersymmetric theories, deformed to $\mathcal{N}=1$ by making the cubic superpotential coupling, $y$, differ from the gauge coupling, $g$, have RG flows somewhat similar to, but crucially different from, asymptotic safety. Setting either $y=0$ or $g=0$, the other coupling is marginally irrelevant. With both $y \neq 0$ and $g \neq 0$, there are RG flows with an IR-attractive line of interacting SCFTs for all $g=y$; the UV limit of the flows for $g \neq y$ has Landau poles. The adjoint chiral superfields $\Phi_{i=1,2,3}$ have $R\left(\Phi_{i}\right)=2 / 3$ for all $g=y$ SCFTs (the $\Phi_{i}$ are not free, because they are not gauge invariant). These examples do not contradict our general arguments. Their one-loop beta function in (5.2) are zero, and that there is a line of fixed points, rather than a RG flow, between the interacting $(g=y \neq 0)$ and free $(g=y=0)$ theories, all with equal value of $a$. And there is no UV SCFT, only IR SCFTs. There are many similar $\mathcal{N}=1$ SCFT examples, along the lines of [47].

Another example of a non-asymptotically free theory is $\mathcal{N}=1$ gauge theory with the three adjoints of $\mathcal{N}=4$, plus $N_{f}$ flavors of matter in the fundamental (as is sometimes considered in the context of AdS/CFT, via added D7 branes). By our general argument, such theories cannot ${ }^{1}$ have a UV-safe SCFT without violating the a-theorem.

\section{Conclusions}

We investigated the nonperturbative gauge dynamics of $\mathcal{N}=1$ super QCD both with, and without, gauge singlet (dual meson) fields $H$, in the $N_{f}, N_{c}$ regime where asymptotic freedom is lost. Unlike the non-supersymmetric case [1], the $H$ fields do not help to achieve asymptotic safety. Instead, the $H$ fields would necessarily violate a unitarity bound in a hypothetical, asymptotically safe SCFT unless their Yukawa coupling is zero and they are decoupled. We showed that the theory without the $H$ fields also cannot have a UV-safe SCFT, because it would violate the a-theorem. We used a-maximization to show that the same issues arise for general gauge groups and matter content.

We arrive at the interesting conclusion that there is a fundamental obstacle to supersymmetric asymptotic safety. Any sensible, UV completion of unsafe IR theories must include many additional degrees of freedom, e.g. those of an unknown, asymptotically free dual.

Note added in revised version: we would like to thank Steven Martin and James Wells for bringing their relevant paper [51] to our attention. Although there is considerable overlap, our paper contains additional methods - for example, using a-maximization, which had not yet been developed at the time [51] was written. Intriguingly, [51] suggested a possible way to construct superconformal UV fixed point theories via superpotential terms.

\footnotetext{
${ }^{1}$ This fits with the dual gravity analysis in [50]. KI thanks David Mateos for pointing out this reference.
} 
To quote an example from [51], consider $\mathrm{SU}\left(N_{c}\right)$ gauge theory with $N_{f}$ fundamental flavor chiral superfields $Q, \widetilde{Q}$, two adjoints chiral superfields $A_{1}$ and $A_{2}$, and a superpotential $W=A_{1} Q \widetilde{Q}+A_{1}^{3}$. The theory is IR free for $N_{f}>N_{c}$. A hypothetical UV interacting fixed point would have $R\left(A_{1}\right)=R(Q)=R(\widetilde{Q})=2 / 3$, and $R\left(A_{2}\right)=\left(N_{f}+N_{c}\right) / 3 N_{c}$, which would satisfy $a_{\mathrm{UV}}>a_{\mathrm{IR}}$ if $R\left(A_{2}\right)>5 / 3$, i.e. if $N_{f}>4 N_{c}$. We subjected this example to a few additional consistency conditions, including those in this paper and also e.g. verifying that $a / c$ satisfies the inequalities of [52]. We have not yet found any inconsistency to definitively rule out the hypothetical UV-safe SCFT for $N_{f}>4 N_{c}$. We note however that replacing the adjoint $A_{1}$ with a gauge singlet $S_{1}$ would not give an interacting SCFT: in that case, since $S_{1}$ is a gauge invariant chiral operator, $R\left(S_{1}\right)=2 / 3$ would imply $\Delta\left(S_{1}\right)=1$ and then $S_{1}$ would necessarily be a free-field, incompatible with the superpotential coupling having an interacting fixed point of its beta function.

\section{Acknowledgments}

The $\mathrm{CP}^{3}$-Origins centre is partially funded by the Danish National Research Foundation, grant number DNRF90. KI is supported in part by the DOE grant DE-SC0009919.

Open Access. This article is distributed under the terms of the Creative Commons Attribution License (CC-BY 4.0), which permits any use, distribution and reproduction in any medium, provided the original author(s) and source are credited.

\section{References}

[1] D.F. Litim and F. Sannino, Asymptotic safety guaranteed, JHEP 12 (2014) 178 [arXiv: 1406.2337] [INSPIRE].

[2] C. Pica and F. Sannino, UV and IR zeros of gauge theories at the four loop order and beyond, Phys. Rev. D 83 (2011) 035013 [arXiv: 1011.5917] [INSPIRE].

[3] F. Sannino and I.M. Shoemaker, Asymptotically safe dark matter, Phys. Rev. D 92 (2015) 043518 [arXiv:1412.8034] [InSPIRE].

[4] N.G. Nielsen, F. Sannino and O. Svendsen, Inflation from asymptotically safe theories, Phys. Rev. D 91 (2015) 103521 [arXiv:1503.00702] [InSPIRE].

[5] D.F. Litim, M. Mojaza and F. Sannino, Vacuum stability of asymptotically safe gauge-Yukawa theories, arXiv:1501.03061 [INSPIRE].

[6] D.H. Rischke and F. Sannino, Thermodynamics of asymptotically safe theories, Phys. Rev. D 92 (2015) 065014 [arXiv: 1505.07828] [INSPIRE].

[7] D.J. Gross and F. Wilczek, Ultraviolet behavior of nonabelian gauge theories, Phys. Rev. Lett. 30 (1973) 1343 [InSPIRE].

[8] H.D. Politzer, Reliable perturbative results for strong interactions?, Phys. Rev. Lett. 30 (1973) 1346 [INSPIRE].

[9] D.J. Gross and F. Wilczek, Asymptotically free gauge theories. 1, Phys. Rev. D 8 (1973) 3633 [INSPIRE]. 
[10] T.P. Cheng, E. Eichten and L.-F. Li, Higgs phenomena in asymptotically free gauge theories, Phys. Rev. D 9 (1974) 2259 [inSPIRE].

[11] D.J.E. Callaway, Triviality pursuit: can elementary scalar particles exist?, Phys. Rept. 167 (1988) 241 [InSPIRE].

[12] B. Holdom, J. Ren and C. Zhang, Stable asymptotically free extensions (safes) of the standard model, JHEP 03 (2015) 028 [arXiv: 1412.5540] [INSPIRE].

[13] G.F. Giudice, G. Isidori, A. Salvio and A. Strumia, Softened gravity and the extension of the standard model up to infinite energy, JHEP 02 (2015) 137 [arXiv:1412.2769] [INSPIRE].

[14] N. Seiberg, Electric-magnetic duality in supersymmetric nonAbelian gauge theories, Nucl. Phys. B 435 (1995) 129 [hep-th/9411149] [INSPIRE].

[15] K.A. Intriligator and N. Seiberg, Lectures on supersymmetric gauge theories and electric-magnetic duality, Nucl. Phys. Proc. Suppl. 45BC (1996) 1 [hep-th/9509066] [INSPIRE].

[16] G. Mack, All unitary ray representations of the conformal group $\mathrm{SU}(2,2)$ with positive energy, Commun. Math. Phys. 55 (1977) 1 [INSPIRE].

[17] J.L. Cardy, Is there a c theorem in four-dimensions?, Phys. Lett. B 215 (1988) 749 [INSPIRE].

[18] H. Osborn, Derivation of a four-dimensional c theorem, Phys. Lett. B 222 (1989) 97 [INSPIRE].

[19] I. Jack and H. Osborn, Analogs for the c theorem for four-dimensional renormalizable field theories, Nucl. Phys. B 343 (1990) 647 [INSPIRE].

[20] A. Cappelli, G. D'Appollonio, R. Guida and N. Magnoli, On the $c$ theorem in more than two-dimensions, PoS(TMR2000)005 [hep-th/0009119] [INSPIRE].

[21] A. Cappelli, R. Guida and N. Magnoli, Exact consequences of the trace anomaly in four-dimensions, Nucl. Phys. B 618 (2001) 371 [hep-th/0103237] [INSPIRE].

[22] Z. Komargodski and A. Schwimmer, On renormalization group flows in four dimensions, JHEP 12 (2011) 099 [arXiv:1107.3987] [INSPIRE].

[23] Z. Komargodski, The constraints of conformal symmetry on RG flows, JHEP 07 (2012) 069 [arXiv:1112.4538] [INSPIRE].

[24] D. Anselmi, D.Z. Freedman, M.T. Grisaru and A.A. Johansen, Nonperturbative formulas for central functions of supersymmetric gauge theories, Nucl. Phys. B 526 (1998) 543 [hep-th/9708042] [INSPIRE].

[25] D. Anselmi, J. Erlich, D.Z. Freedman and A.A. Johansen, Positivity constraints on anomalies in supersymmetric gauge theories, Phys. Rev. D 57 (1998) 7570 [hep-th/9711035] [INSPIRE].

[26] K.A. Intriligator and B. Wecht, The exact superconformal $R$ symmetry maximizes a, Nucl. Phys. B 667 (2003) 183 [hep-th/0304128] [INSPIRE].

[27] D. Kutasov, New results on the 'a theorem' in four-dimensional supersymmetric field theory, hep-th/0312098 [INSPIRE].

[28] D. Kutasov, A. Parnachev and D.A. Sahakyan, Central charges and U(1)R symmetries in $N=1$ super Yang-Mills, JHEP 11 (2003) 013 [hep-th/0308071] [INSPIRE]. 
[29] K.A. Intriligator and B. Wecht, $R G$ fixed points and flows in $S Q C D$ with adjoints, Nucl. Phys. B 677 (2004) 223 [hep-th/0309201] [INSPIRE].

[30] E. Barnes, K.A. Intriligator, B. Wecht and J. Wright, Evidence for the strongest version of the $4 D$ a-theorem, via a-maximization along RG flows, Nucl. Phys. B 702 (2004) 131 [hep-th/0408156] [INSPIRE].

[31] D. Kutasov and A. Schwimmer, Lagrange multipliers and couplings in supersymmetric field theory, Nucl. Phys. B 702 (2004) 369 [hep-th/0409029] [INSPIRE].

[32] T. Banks and A. Zaks, On the phase structure of vector-like gauge theories with massless fermions, Nucl. Phys. B 196 (1982) 189 [inSPIRE].

[33] O. Antipin, M. Gillioz, E. Mølgaard and F. Sannino, The a theorem for gauge-Yukawa theories beyond Banks-Zaks fixed point, Phys. Rev. D 87 (2013) 125017 [arXiv:1303.1525] [INSPIRE].

[34] M.E. Machacek and M.T. Vaughn, Two loop renormalization group equations in a general quantum field theory. 1. Wave function renormalization, Nucl. Phys. B 222 (1983) 83 [INSPIRE].

[35] M.E. Machacek and M.T. Vaughn, Two loop renormalization group equations in a general quantum field theory. 2. Yukawa couplings, Nucl. Phys. B 236 (1984) 221 [inSPIRE].

[36] M.E. Machacek and M.T. Vaughn, Two loop renormalization group equations in a general quantum field theory. 3. Scalar quartic couplings, Nucl. Phys. B 249 (1985) 70 [INSPIRE].

[37] I. Jack and H. Osborn, General background field calculations with fermion fields, Nucl. Phys. B 249 (1985) 472 [InSPIRE].

[38] A. Hasenfratz, P. Hasenfratz, K. Jansen, J. Kuti and Y. Shen, The equivalence of the top quark condensate and the elementary Higgs field, Nucl. Phys. B 365 (1991) 79 [INSPIRE].

[39] P. Gerhold and K. Jansen, The phase structure of a chirally invariant lattice Higgs-Yukawa model for small and for large values of the Yukawa coupling constant, JHEP 09 (2007) 041 [arXiv:0705.2539] [INSPIRE].

[40] P. Gerhold, K. Jansen and J. Kallarackal, The Higgs boson resonance width from a chiral Higgs-Yukawa model on the lattice, Phys. Lett. B 710 (2012) 697 [arXiv:1111.4789] [INSPIRE].

[41] D.Y.J. Chu, K. Jansen, B. Knippschild, C.J.D. Lin and A. Nagy, A lattice study of a chirally invariant Higgs-Yukawa model including a higher dimensional $\phi^{6}$-term, Phys. Lett. B 744 (2015) 146 [arXiv:1501.05440] [INSPIRE].

[42] S. Catterall and A. Veernala, Four fermion interactions in non-abelian gauge theory, Phys. Rev. D 87 (2013) 114507 [arXiv:1303.6187] [INSPIRE].

[43] S. Ferrara and B. Zumino, Transformation properties of the supercurrent, Nucl. Phys. B 87 (1975) 207 [InSPIRE].

[44] B. Grinstein, K.A. Intriligator and I.Z. Rothstein, Comments on unparticles, Phys. Lett. B 662 (2008) 367 [arXiv:0801.1140] [INSPIRE].

[45] V.A. Novikov, M.A. Shifman, A.I. Vainshtein and V.I. Zakharov, Exact Gell-Mann-Low function of supersymmetric Yang-Mills theories from instanton calculus, Nucl. Phys. B 229 (1983) 381 [inSPIRE]. 
[46] A.B. Zamolodchikov, Irreversibility of the flux of the renormalization group in a $2 D$ field theory, JETP Lett. 43 (1986) 730 [Pisma Zh. Eksp. Teor. Fiz. 43 (1986) 565] [inSPIRE].

[47] R.G. Leigh and M.J. Strassler, Exactly marginal operators and duality in four-dimensional $N=1$ supersymmetric gauge theory, Nucl. Phys. B 447 (1995) 95 [hep-th/9503121] [INSPIRE].

[48] K.A. Intriligator, IR free or interacting? A proposed diagnostic, Nucl. Phys. B 730 (2005) 239 [hep-th/0509085] [INSPIRE].

[49] A. Amariti and K. Intriligator, $(\Delta a)$ curiosities in some $4 D S U S Y$ RG flows, JHEP 11 (2012) 108 [arXiv: 1209.4311] [InSPIRE].

[50] E. D'Hoker and Y. Guo, Rigidity of $\mathrm{SU}(2,2 \mid 2)$-symmetric solutions in type IIB, JHEP 05 (2010) 088 [arXiv: 1001.4808] [INSPIRE].

[51] S.P. Martin and J.D. Wells, Constraints on ultraviolet stable fixed points in supersymmetric gauge theories, Phys. Rev. 64 (2001) 036010 [hep-ph/0011382] [INSPIRE].

[52] D.M. Hofman and J. Maldacena, Conformal collider physics: energy and charge correlations, JHEP 05 (2008) 012 [arXiv:0803.1467] [INSPIRE]. 